\title{
Partial liquidation under reference-dependent preferences
}

\author{
Vicky Henderson ${ }^{1} \cdot$ Jonathan $_{\text {Muscat }}{ }^{1}$
}

Received: 7 April 2018 / Accepted: 3 November 2019 / Published online: 16 March 2020

(C) The Author(s) 2020

\begin{abstract}
We propose a multiple optimal stopping model where an investor can sell a divisible asset position at times of her choosing. Investors have $S$-shaped referencedependent preferences, whereby utility is defined over gains and losses relative to a reference level and is concave over gains and convex over losses. For a price process following a time-homogeneous diffusion, we employ the constructive potentialtheoretic solution method developed by Dayanik and Karatzas (Stoch. Process. Appl. 107:173-212, 2003). As an example, we revisit the single optimal stopping model of Kyle et al. (J. Econ. Theory 129:273-288, 2006) to allow partial liquidation. In contrast to the extant literature, we find that the investor may partially liquidate the asset at distinct price thresholds above the reference level. Under other parameter combinations, the investor sells the asset in a block, either at or above the reference level.
\end{abstract}

Keywords Multiple optimal stopping - Reference-dependent preferences · Prospect theory $\cdot$ Behavioural finance

Mathematics Subject Classification (2010) $60 \mathrm{G} 40 \cdot 60 \mathrm{~J} 60$

JEL Classification D81 - G40

Jonathan Muscat is supported by a Leverhulme Trust Doctoral Scholarship via the "Bridges" programme at the University of Warwick.

$凶$ V. Henderson

vicky.henderson@warwick.ac.uk

J. Muscat

j.muscat@warwick.ac.uk

1 Department of Statistics, University of Warwick, Coventry CV4 7AL, UK 


\section{Introduction}

Prospect theory was proposed by Kahneman and Tversky [22] and extended by Tversky and Kahneman [33]. Under prospect theory, utility is reference-dependent and so is defined over gains and losses relative to a reference level, rather than over final wealth. The utility function exhibits concavity in the domain of gains and convexity in the domain of losses, and the function is steeper for losses than for gains, a feature known as loss aversion. Prospect theory was originally developed to better fit decision making behaviour observed in experimental studies.

In recent years, optimal stopping models employing reference-dependent preferences have been developed in order to understand the dynamic behaviour of individuals with such preferences and to see to what extent the theory can be used to explain both experimental and empirically observed behaviour. A strand of this literature, beginning with Kyle et al. [24], has considered problems of optimal sale timing of risky assets under reference-dependent preferences. In this paper, we extend the model of Kyle et al. [24] to consider the question of partial liquidation of assets. Indeed, [24, p. 284] remark that "... it would be of interest to incorporate partial liquidation in our model".

We propose an infinite-horizon optimal stopping model where an investor with $S$-shaped reference-dependent preferences can sell her divisible asset position at times of her choosing in the future. She derives utility from gains and losses relative to a reference level, and utility is realised at the time she sells her last tranche of asset. We first give a general result which allows a multiple stopping problem (where stopping times are allowed to coincide) to be viewed as a sequence of standard optimal stopping problems. This result is then applied to a model where utility is given by piecewise exponential functions, steeper for losses than for gains, and the asset price follows a Brownian motion with drift. These explicit calculations enable us to compare to the paper of Kyle et al. [24] who solve the block-sale case under the same modelling assumptions.

Our main finding is showing that in the extended Kyle et al. [24] model, the investor may engage in partial sales. This represents the first time it has been shown that partial liquidation can occur under an $S$-shaped value function. Decisions depend upon the Sharpe ratio of the asset together with the risk aversion, risk seeking and loss aversion measures. We give a condition on parameters which distinguishes whether an investor will sell at two distinct thresholds (partial sales) or sell both units at a single threshold (block sale). In some circumstances, the investor liquidates at exactly break-even, which captures the spirit of the break-even effect of Thaler and Johnson [32]. In an experimental setting, the authors of [32] find that in the presence of prior losses, gambles that offer a chance to break even are very attractive. There are also parameter combinations which lead to the investor holding the asset indefinitely or, in the other extreme, sell everything immediately. Finally, there is a parameter regime where if the investor were restricted to block sales, she would not enter the game. However, for the same parameter set, when partial sales are allowed, she will enter and wait to sell both units of asset at a gain.

Researchers are interested in modelling investor trading behaviour under $S$-shaped reference-dependent preferences (of prospect theory) to see if it can better explain 
stylised facts in the empirical and experimental data. In particular, reference-dependence is a long-standing explanation of why individual investors tend to sell winners too early and ride losers too long, a behaviour called the disposition effect (Shefrin and Statman [31], Odean [28]). In this vein, Kyle et al. [24], Henderson [14], Barberis and Xiong [4] and Ingersoll and Jin [20] contribute optimal stopping models for an investor with reference-dependent preferences under differing assumptions. Kyle et al. [24] and Henderson [14] treat one-shot or block-sale optimal stopping problems under alternative assumptions on the $S$-shaped utility and price processes. In particular, Henderson [14] (see also Ingersoll and Jin [20]) contributed a model where the investor sells at a loss voluntarily. This provided a better match to the disposition effect. Henderson [14] also considers partial liquidation, but finds under the Kahneman-Tversky $S$-shaped value function and exponential Brownian motion that the agent did not choose to partially sell.

Evidence for reference-dependent loss aversion has been found using slot machine gambler's decisions by Lien and Zheng [26]. Laboratory experiments of Magnani [27] find that individual investors sell winners too early and ride losers too long. Imas [19] studies how realised and paper losses affect behaviour in an experiment where subjects make a sequence of investment decisions. In one of the treatments of this experiment, subjects decide whether to realise the outcome of the investment in the middle of the sequence and are found to be more likely to realise gains than losses.

Barberis and Xiong [4], Ingersoll and Jin [20] (and also He and Yang [13]) consider realisation utility models where investors treat their investing experience as a series of investment episodes and receive utility from each individual sale at the time of sale. Mathematically, they sum up the utility of each individual sale and use a discount factor to model investors' tendency to realise gains early and losses late. Barberis and Xiong [4] assume a piecewise linear utility function and find that the investors never voluntarily sell a stock at a loss. Ingersoll and Jin [20] extend the model by assuming an $S$-shaped utility function and find that the investors voluntarily sell a stock both at a gain and at a loss. Recently, He and Yang [13] extended the model to include an adaptive reference point which adapts to the stock's prior gain or loss. However, each of these models is separable in the sense that multiple identical units of assets would be sold simultaneously at the same threshold. None address the question of partial liquidation.

Our aim in this paper is to give a simple, tractable optimal stopping model with $S$-shape reference-dependent preferences where partial sales $d o$ arise as an optimal behaviour. We employ the constructive potential-theoretic solution method developed by Dayanik and Karatzas [9] for optimal stopping of linear diffusions. This approach is particularly useful for our problem as the smooth-fit principle does not apply because of the non-differentiability of the utility function, making the usual variational approach more challenging to apply. One-dimensional optimal stopping problems have been analysed by exploiting the relationship between functional concavity and $r$-excessivity (see Dynkin and Yushkevich [10, Chap. 3, Sect. 8]) which has been applied by Dayanik and Karatzas [9]. See also Alvarez [1, 2] for related techniques. A general approach for multiple optimal stopping is presented in Kobylanski et al. [23], and our separation of our original problem into $N$ optimal stopping 
sub-problems is in the same spirit. We provide a direct construction and proof in our setting as we have a natural ordering on the stopping times. Other work on multiple optimal stopping has appeared in Carmona and Dayanik [7] who consider a problem for a regular diffusion process in the context of American options when the holder has a number of exercise rights. It is assumed there that the holder chooses the consecutive stopping times with a strictly positive break period called a random refraction period (otherwise the holder would use all her rights at the same time). It is difficult to explicitly determine the solution, and the authors of [7] describe a recursive algorithm. The recent work of Christensen [8] offers an alternative approach to multiple stopping with random refraction periods as a special case of a class of impulse control problems and employs harmonic function techniques. In contrast, in our problem here, we do not wish to impose any breaks between stopping times, but rather formulate a model setting where it may be optimal to have such breaks. Finally, direct methods for optimal stopping have also been used in stochastic switching problems (Bayraktar and Egami [5]), and similar ideas are employed by Henderson and Hobson [15] to solve a problem involving a perfectly divisible tranche of options on an asset with a diffusion price process.

One strand of the recent literature has concerned itself with portfolio optimisation (optimal control) under prospect theory, and examples of this work include Jin and Zhou [21] and Carassus and Rásonyi [6]. Another focus of the recent literature is on the probability weighting of prospect theory. However, probability weighting leads to a time-inconsistency and thus a difference in behaviour of naive and sophisticated agents; see Barberis [3]. Henderson et al. [17] (building on seminal work of Xu and Zhou [34]) study agents who can pre-commit to a strategy and show that under some assumptions (satisfied by the models of interest including the Kahneman and Tversky [22, 33] specification), this consists of a stop-loss threshold together with a continuous distribution on gains. However, recent results (Ebert and Strack [11], also Henderson et al. [16]) have shown that naive prospect theory agents never stop gambling. We focus in this paper on reference-dependent $S$-shaped preferences in the absence of probability weighting and extend the literature in the direction of holding a quantity of asset rather than just one unit.

\section{General framework}

\subsection{The partial liquidation problem}

Consider an investor who is holding $N \geq 1$ units of claim on an asset with current price $Y_{t}$, where we denote by $\mathbb{F}=\left(\mathcal{F}_{t}\right)$ the natural filtration of $Y$. The investor is able to liquidate or sell the position in the asset at any time in the future. She can choose $\mathbb{F}$-stopping times $\tau_{i}, i=1, \ldots, N$, at which to liquidate her $N$ units of the claim, and hence is able to partially liquidate her divisible position. We write $\tau_{1} \geq \cdots \geq \tau_{N}$ so that $\tau_{i}$ denotes the sale time when there are $i$ units remaining in the portfolio. For each sold unit $i$, the investor receives the payoff $h^{i}\left(Y_{\tau_{i}}\right)$, where the $h^{i}(\cdot)$ are nondecreasing functions, and compares this amount to a corresponding reference level $h_{R}^{i}$. As is often the case in the literature, an interpretation of $h_{R}^{i}$ is the break-even level or the amount paid for the claim on the asset itself, and we later specialise to this choice. 
We should like a formulation in which the potential partial sales are not independent (so delaying a partial sale will impact on future sales), and so our investor considers her position as an investment episode which is closed and valued only once the final partial sale takes place. This might be appropriate for institutional investors who are more likely to view investments in terms of overall portfolio position. Under this interpretation, the investor's problem can be written as

$$
V_{N}(y, 0)=\sup _{\tau_{1} \geq \cdots \geq \tau_{N}} \mathbb{E}\left[U\left(\sum_{i=1}^{N}\left(h^{i}\left(Y_{\tau_{i}}\right)-h_{R}^{i}\right)\right) \mid Y_{0}=y\right],
$$

where the utility function $U$ is an increasing function. Later we specialise to the reference-dependent $S$-shaped function $U$ given in the next section. Furthermore, we set $f\left(Y_{\tau}(\omega)\right)=0$ on $\{\tau=+\infty\}$ for any Borel function $f(\cdot)$.

While later we assume a linear payoff function for each partial sale, i.e., $h^{i}(y)=y$ for all $i$, the methodology can be used to treat more complex payoffs. For example, take $N=2$ and call options $h^{1}(y)=\left(y-k_{1}\right)^{+}, h^{2}(y)=\left(y-k_{2}\right)^{+}$with strikes $k_{1}>k_{2}$. Denote by $h_{R}^{1}, h_{R}^{2}$ two different reference levels, with one interpretation being the price paid for each option. Using a general ordering result in Henderson et al. [18], we know that the options are exercised in increasing strike order, and hence our solution method applies.

Finally, to close this section, we comment on alternative specifications. First note that in common with Kyle et al. [24], we do not include a discount term in the specification above. Although it would be possible to do so, it is questionable whether this is desirable when losses are involved. Discounting gives an incentive to stop sooner on gains but delay losses, a feature which we, and others, are trying to explain by using $S$-shaped reference-dependent preferences. Second, as shown in Henderson [14] and Barberis and Xiong [4], if the investor instead considered each partial sale as an independent investment episode, then she would consider

$$
\sup _{\tau_{1} \geq \cdots \geq \tau_{N}} \mathbb{E}\left[\sum_{i=1}^{N} U\left(h^{i}\left(Y_{\tau_{i}}\right)-h_{R}^{i}\right) \mid Y_{0}=y\right] .
$$

While this captures the spirit of Barberis and Xiong's [4] realisation utility where investors consider a series of investing 'episodes', mathematically, this formulation splits into $N$ independent stopping problems and thus does not capture the interdependency we desire.

\subsection{Reference-dependent preferences}

When we present results for a specific model, we take the $S$-shaped, two-piece exponential utility function used by Kyle et al. [24], i.e.,

$$
U(x)= \begin{cases}\phi_{1}\left(1-e^{-\gamma_{1} x}\right), & \text { if } x \geq 0, \\ \phi_{2}\left(e^{\gamma_{2} x}-1\right), & \text { if } x<0,\end{cases}
$$

where $\phi_{1}, \phi_{2}, \gamma_{1}, \gamma_{2}>0$. Above the reference point, the agent's utility function is a concave exponential function, with $\gamma_{1}$ measuring the local absolute risk aversion. 
Below the reference point, the value function is a convex exponential function, with $\gamma_{2}$ measuring the local absolute risk loving level. In addition, we assume $\phi_{1} \gamma_{1}<\phi_{2} \gamma_{2}$ to ensure that the agent is loss averse, that is, more sensitive to losses than to gains around the reference point, i.e., $U^{\prime}(0-)>U^{\prime}(0+)$.

\subsection{The price process}

Consider a complete probability space $(\Omega, \mathcal{F}, \mathbb{F}, \mathbb{P})$ supporting a Brownian motion $W=\left(W_{t}\right)_{t \geq 0}$ and let $Y=\left(Y_{t}\right)_{t \geq 0}$ be a one-dimensional time-homogeneous diffusion process solving

$$
d Y_{t}=\mu\left(Y_{t}\right) d t+\sigma\left(Y_{t}\right) d W_{t}
$$

for Borel functions ${ }^{1} \mu: \mathcal{I} \rightarrow \mathbb{R}$ and $\sigma: \mathcal{I} \rightarrow \mathbb{R}_{+}$, where $\mathcal{I}=\left(a_{\mathcal{I}}, b_{\mathcal{I}}\right) \subseteq \mathbb{R}$ is the state space of $Y$ with endpoints $-\infty \leq a_{\mathcal{I}}<b_{\mathcal{I}} \leq \infty$. Assume $Y$ is regular in $\left(a_{\mathcal{I}}, b_{\mathcal{I}}\right)$. Let $\tau_{(a, b)}^{Y}=\inf \left\{t: Y_{t} \notin(a, b)\right\}$ be the first time $Y$ leaves the interval $(a, b)$. Consider the continuous, strictly increasing scale function $S(\cdot)$ of the diffusion $Y$, satisfying

$$
\mathcal{D} S(x):=\frac{1}{2} \sigma^{2}(x) \frac{d^{2} S}{d x^{2}}(x)+\mu(x) \frac{d S}{d x}(x)=0, \quad x \in \mathcal{I},
$$

and ensuring that the process $S\left(Y_{t \wedge \tau_{\left(a_{\mathcal{I}}, b_{\mathcal{I}}\right)}^{Y}}\right)$ is a local martingale; see Revuz and Yor [30, Proposition VII.3.5].

Specifically, we specialise to the model used in Kyle et al. [24] and hence take

$$
d Y_{t}=\mu d t+\sigma d W_{t},
$$

where $\mu$ and $\sigma>0$ are constants and $\mathcal{I}=(-\infty, \infty)$. Under these assumptions, the scale function is given by $S(y)=e^{\eta y}$ if $\mu<0,-e^{\eta y}$ if $\mu>0$ and $S(y)=y$ if $\mu=0$, where $\eta=-2 \mu / \sigma^{2}$. Note that $\eta$ depends solely on the return-for-risk ratio or Sharpe ratio $\mu / \sigma^{2}$.

\section{Solution to the partial liquidation problem}

\subsection{The general problem}

An approach towards solving the optimal stopping problem in (2.1) is outlined in Kobylanski et al. [23]. This approach breaks down the original optimal stopping problem into $N$ sub-problems. In Proposition 3.1 below, we provide an alternative construction and proof of how such a decomposition can be achieved. However, it is worth noting that the result is in the same spirit as the discussion presented in Kobylanski et al. [23], particularly Theorem 3.1 there.

\footnotetext{
${ }^{1}$ We assume that $\mu(\cdot)$ and $\sigma(\cdot)$ are sufficiently regular so that there exists a weak solution to the SDE which is unique in the sense of probability law, so that the scale function $S(\cdot)$ exists. See Revuz and Yor [30, Proposition VII.3.2].
} 
Denote by $x$ the total gains or losses from previous sales. Define

$$
\begin{aligned}
V_{N}(y, x) & =\sup _{\tau_{1} \geq \cdots \geq \tau_{N}} \mathbb{E}\left[U\left(x+\sum_{i=1}^{N}\left(h^{i}\left(Y_{\tau_{i}}\right)-h_{R}^{i}\right)\right) \mid Y_{0}=y\right] \\
& =\sup _{\tau_{1} \geq \cdots \geq \tau_{N}} \mathbb{E}\left[\mathbb{E}\left[U\left(x+\sum_{i=1}^{N}\left(h^{i}\left(Y_{\tau_{i}}\right)-h_{R}^{i}\right)\right) \mid \mathcal{F}_{\tau_{N}}\right] \mid Y_{0}=y\right] .
\end{aligned}
$$

We are primarily interested in (2.1), i.e., $x=0$. The following result will facilitate the decomposition of (3.1) into $N$ sub-problems.

In order to be able to solve (3.1), we assume that the usual integrability condition holds (see for example Peskir and Shiyaev [29, Eq. (2.1.1)] for the one-dimensional case, or Kobylanski et al. [23, Theorem 2.3]), namely

$$
\mathbb{E}\left[\sup _{0 \leq t_{N} \leq \cdots \leq t_{1}<\infty}\left|U\left(x+\sum_{i=1}^{N}\left(h^{i}\left(Y_{t_{i}}\right)-h_{R}^{i}\right)\right)\right|\right]<\infty .
$$

Proposition 3.1 Let $(\Omega, \mathcal{F}, \mathbb{P})$ be a probability space, $Y$ a time-homogeneous diffusion process with respect to its natural filtration $\mathbb{F}$ and $f(\cdot)$ any increasing continuous function with $f(0)=0$. If

$$
\mathbb{E}\left[\sup _{0 \leq t_{n} \leq \cdots \leq t_{1}<\infty}\left|f\left(\sum_{i=1}^{n} h^{i}\left(Y_{t_{i}}\right)\right)\right|\right]<\infty
$$

then

$$
\begin{aligned}
& \sup _{\tau_{n} \leq \cdots \leq \tau_{1}} \mathbb{E}\left[\mathbb{E}\left[f\left(\sum_{i=1}^{n} h^{i}\left(Y_{\tau_{i}}\right)\right) \mid \mathcal{F}_{\tau_{n}}\right] \mid Y_{0}=y\right] \\
& \quad=\sup _{\tau_{n}} \mathbb{E}\left[\underset{\tau_{n-1} \leq \cdots \leq \tau_{1}: \tau_{n-1} \geq \tau_{n}}{\operatorname{ess} \sup } \mathbb{E}\left[f\left(\sum_{i=1}^{n} h^{i}\left(Y_{\tau_{i}}\right)\right) \mid \mathcal{F}_{\tau_{n}}\right] \mid Y_{0}=y\right] .
\end{aligned}
$$

Proof The result follows if we show that

$$
\begin{aligned}
& \sup _{\tau_{n} \leq \cdots \leq \tau_{1}} \mathbb{E}\left[\mathbb{E}\left[f\left(\sum_{i=1}^{n} h^{i}\left(Y_{\tau_{i}}\right)\right) \mid \mathcal{F}_{\tau_{n}}\right] \mid Y_{0}=y\right] \\
& \geq \sup _{\tau_{n}} \mathbb{E}\left[\operatorname{esssup}_{\tau_{n-1} \leq \cdots \leq \tau_{1}: \tau_{n-1} \geq \tau_{n}} \mathbb{E}\left[f\left(\sum_{i=1}^{n} h^{i}\left(Y_{\tau_{i}}\right)\right) \mid \mathcal{F}_{\tau_{n}}\right] \mid Y_{0}=y\right]
\end{aligned}
$$

since the reverse inequality is trivial. Given an arbitrary stopping time $\tau_{n}$, consider the random variable

$$
Z^{\left(\tau_{n-1}, \ldots, \tau_{1}\right)}:=\mathbb{E}\left[f\left(\sum_{i=1}^{n} h^{i}\left(Y_{\tau_{i}}\right)\right) \mid \mathcal{F}_{\tau_{n}}\right]
$$


and the family $\Gamma=\left\{Z^{\alpha}: \alpha \in \mathcal{I}\right\}$, where $\mathcal{I}$ is the set of all $(n-1)$-tuples of $\mathbb{F}$-stopping times $\left(\xi_{n-1}, \ldots, \xi_{1}\right)$ satisfying $\tau_{n} \leq \xi_{n-1} \leq \cdots \leq \xi_{1}$ almost surely. As shown in Lemma A.1, the family $\Gamma$ has the lattice property and hence there exists a countable subset $\mathcal{J}=\left\{\alpha^{j}: j \in \mathbb{N}\right\} \subseteq \mathcal{I}$ such that

$$
Z^{*}:=\underset{\alpha \in \mathcal{I}}{\operatorname{esssup}} Z^{\alpha}=\lim _{j \rightarrow \infty} Z^{\alpha^{j}} \quad \text { with } Z^{\alpha^{1}} \leq Z^{\alpha^{2}} \leq \cdots, \mathbb{P} \text {-a.s. }
$$

It follows by using (3.3) and Jensen's inequality that

$$
\begin{aligned}
\mathbb{E}\left[\left|Z^{*}\right|\right] & =\mathbb{E}\left[\left|\lim _{j \rightarrow \infty} Z^{\alpha^{j}}\right|\right]=\mathbb{E}\left[\lim _{j \rightarrow \infty}\left|Z^{\alpha^{j}}\right|\right] \\
& =\mathbb{E}\left[\lim _{j \rightarrow \infty}\left|\mathbb{E}\left[f\left(h^{n}\left(Y_{\tau_{n}}\right)+\sum_{i=1}^{n-1} h^{i}\left(Y_{\alpha_{i}^{j}}\right)\right) \mid \mathcal{F}_{\tau_{n}}\right]\right|\right] \\
& \leq \mathbb{E}\left[\lim _{j \rightarrow \infty} \mathbb{E}\left[\left|f\left(h^{n}\left(Y_{\tau_{n}}\right)+\sum_{i=1}^{n-1} h^{i}\left(Y_{\alpha_{i}^{j}}\right)\right)\right| \mid \mathcal{F}_{\tau_{n}}\right]\right] \\
& \leq \mathbb{E}\left[\mathbb{E}\left[\operatorname{ess~sup}_{\left(\xi_{n-1}, \ldots, \xi_{1}\right) \in \mathcal{I}}\left|f\left(h^{n}\left(Y_{\tau_{n}}\right)+\sum_{i=1}^{n-1} h^{i}\left(Y_{\xi_{i}}\right)\right)\right| \mid \mathcal{F}_{\tau_{n}}\right]\right] \\
& \leq \mathbb{E}\left[\sup _{t_{n} \leq \cdots \leq t_{1}}\left|f\left(\sum_{i=1}^{n} h^{i}\left(Y_{t_{i}}\right)\right)\right|\right]<\infty .
\end{aligned}
$$

Hence by monotone convergence, the right-hand side of (3.4) becomes $\sup _{\tau_{n}} \mathbb{E}\left[\operatorname{ess~sup}_{\alpha \in \mathcal{I}} Z^{\alpha}\right]=\sup _{\tau_{n}} \lim _{j \rightarrow \infty} \mathbb{E}\left[Z^{\alpha^{j}}\right] \leq \sup _{\tau_{n}} \sup _{\tau_{n-1} \leq \ldots \leq \tau_{1}: \tau_{n-1} \geq \tau_{n}} \mathbb{E}\left[Z^{\left(\tau_{n-1}, \ldots, \tau_{1}\right)}\right]$.

Assuming that condition (3.2) applies, Proposition 3.1 implies that for $1 \leq n \leq N$,

$$
\begin{aligned}
V_{n}(y, x) & =\sup _{\tau_{n} \leq \cdots \leq \tau_{1}} \mathbb{E}\left[U\left(x+\sum_{i=1}^{n}\left(h^{i}\left(Y_{\tau_{i}}\right)-h_{R}^{i}\right)\right) \mid Y_{0}=y\right] \\
& =\sup _{\tau_{n}} \mathbb{E}\left[\sup _{\tau_{n-1} \leq \cdots \leq \tau_{1}: \tau_{n-1} \geq \tau_{n}} \mathbb{E}\left[U\left(x+\sum_{i=1}^{n}\left(h^{i}\left(Y_{\tau_{i}}\right)-h_{R}^{i}\right)\right) \mid \mathcal{F}_{\tau_{n}}\right] \mid Y_{0}=y\right] \\
& =\sup _{\tau_{n}} \mathbb{E}\left[V_{n-1}\left(Y_{\tau_{n}}, x+h^{n}\left(Y_{\tau_{n}}\right)-h_{R}^{n}\right) \mid Y_{0}=y\right],
\end{aligned}
$$

where $V_{0}(y, x)=U(x)$.

Given the time-homogeneity of the problem, the structure of the solution must be to stop when the price process $Y$ exits an interval. Thus the approach is to consider stopping times of this form and choose the 'best' interval. We first transform the problem into natural scale-this simplifies calculations as we then work with (local) martingales. Define $\Theta_{t}=S\left(Y_{t}\right)$ to transform the process into natural scale and let 
$\Theta_{0}=\theta_{0}=S\left(Y_{0}\right)$. We can map exit times of the price $Y$ from an interval to exit times of $\Theta$ from a transformed interval, i.e.,

$$
\begin{aligned}
\tau_{(a, b)}^{Y} & =\inf \left\{t: Y_{t} \notin(a, b)\right\}=\inf \left\{t: \Theta_{t} \notin(S(a), S(b))\right\} \\
& =\inf \left\{t: \Theta_{t} \notin(\phi, \psi)\right\}=\tau_{(\phi, \psi)}^{\Theta},
\end{aligned}
$$

where $S(a)=\phi, S(b)=\psi$.

Define $g_{n}(\theta, x)$ to be the value of the problem with initially $1 \leq n \leq N$ units remaining, current gains and losses $x$ from previous sales, current value of the transformed price $\theta$, and sell one unit immediately so that $n-1$ remain. Then by definition,

$$
g_{n}(\theta, x)=V_{n-1}\left(S^{-1}(\theta), x+h^{n}\left(S^{-1}(\theta)\right)-h_{R}^{n}\right) .
$$

We now give some intuitive arguments to describe the solution when we consider a bounded interval and then follow this with more cases in Proposition 3.2. Consider first any fixed interval $(a, b) \in \mathcal{I}$ such that $(S(a), S(b))$ is bounded, and with $n$ units remaining, compute

$$
\begin{aligned}
& \mathbb{E}\left[V_{n-1}\left(Y_{\tau_{(a, b)}^{Y}}, x+h^{n}\left(Y_{\tau_{(a, b)}^{Y}}\right)-h_{R}^{n}\right) \mid Y_{0}=y\right]=\mathbb{E}\left[g_{n}\left(\Theta_{\tau_{(\phi, \psi)}^{\Theta}}, x\right) \mid \Theta_{0}=\theta\right] \\
& =g_{n}(\phi, x) \frac{\psi-\theta}{\psi-\phi}+g_{n}(\psi, x) \frac{\theta-\phi}{\psi-\phi},
\end{aligned}
$$

where we use the probabilities of the (bounded) martingale $\left(\Theta_{t}\right)_{t \leq \tau_{(\phi, \psi)}^{\Theta}}$ hitting the ends of the interval. We then choose the 'best' interval $(\phi, \psi)$, i.e., consider

$$
\sup _{\phi<\theta<\psi}\left(g_{n}(\phi, x) \frac{\psi-\theta}{\psi-\phi}+g_{n}(\psi, x) \frac{\theta-\phi}{\psi-\phi}\right)=\bar{g}_{n}(\theta, x),
$$

where $\bar{g}_{n}(\cdot, x)$ is the smallest concave majorant of the function $g_{n}(\cdot, x)$ for any fixed $x$.

Figure 1 gives a stylised representation of $\theta \mapsto g_{n}(\theta, x)$ for fixed $x$. We can use the graph to explain intuitively why the solution to the 'best' interval problem above is indeed the smallest concave majorant. We want to choose endpoints $\phi, \psi$ to maximise the expression in brackets in (3.6). If we start at the point $\theta_{A}$ on the graph, then the expression in the curly brackets in (3.6) is maximised by taking $\phi=\psi=\theta_{A}$ (all other pairs of endpoints give values beneath $\left.g_{n}\left(\theta_{A}, x\right)\right)$. This corresponds to immediate stopping since we stop when we exit the interval $(\phi, \psi)$. However, if we start at the point $\theta_{B}$, the quantity in brackets is maximised if we take $\phi=\phi_{B}$ and $\psi=\psi_{B}$. In fact, for any starting point in the interval $\left(\phi_{B}, \psi_{B}\right)$, the endpoints $\phi_{B}, \psi_{B}$ are best. Thus, for any $\theta \in\left(\phi_{B}, \psi_{B}\right)$, the solution is to stop when the transformed price $\Theta$ reaches either endpoint of the interval. Outside the interval $\left(\phi_{B}, \psi_{B}\right)$, the solution is to stop immediately. So the solution is the smallest concave majorant, which is equal to the function $g_{n}$ itself for $\theta$ outside the interval $\left(\phi_{B}, \psi_{B}\right)$ and the dashed straight line joining the endpoints for values of $\theta$ inside the interval. This intuition lies behind the following result. 
Fig. 1 Stylised representation of the function $\theta \mapsto g_{n}(\theta, x)$ for fixed $x$, where $\theta=S(y)$. The function $g_{n}(\cdot, x)$ represents the value of the problem to the investor holding $1 \leq n \leq N$ units of asset if she sells immediately. The smallest concave majorant $\bar{g}_{n}(\cdot, x)$ is formed by taking the straight dashed line for $\theta \in\left(\phi_{B}, \psi_{B}\right)$ and the function $g_{n}(\cdot, x)$ itself for $\theta \leq \phi_{B}$ and $\theta \geq \psi_{B}$.

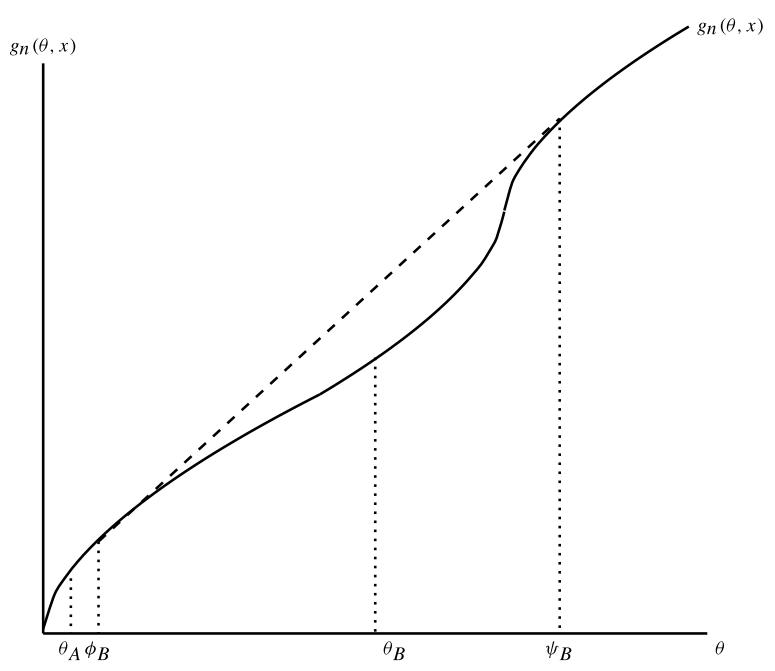

Proposition 3.2 Consider the optimal stopping problem defined in (2.1) with $U(\cdot)$ an increasing bounded function, $Y=\left(Y_{t}\right)_{t \geq 0}$ a one-dimensional time-homogeneous diffusion process with dynamics given in (2.3) and state space $\mathcal{I}=\left(a_{\mathcal{I}}, b_{\mathcal{I}}\right) \subseteq \mathbb{R}$ in which $Y$ is regular. On the interval $\left(S\left(a_{\mathcal{I}}\right), S\left(b_{\mathcal{I}}\right)\right)$, let $\bar{g}_{n}(\cdot, x)$ be the smallest concave majorant of $g_{n}(\cdot, x)$ for any fixed $x$, where $1 \leq n \leq N$.

(i) If $S\left(a_{\mathcal{I}}\right)=-\infty$, then $V_{n}(y, x)=V_{n-1}\left(b_{\mathcal{I}}, x+h^{n}\left(b_{\mathcal{I}}\right)-h_{R}^{n}\right)$ for $y \in\left(a_{\mathcal{I}}, b_{\mathcal{I}}\right)$.

(ii) If $S\left(a_{\mathcal{I}}\right)>-\infty$, then $V_{n}(y, x)=\bar{g}_{n}(S(y), x)$ for $y \in\left(a_{\mathcal{I}}, b_{\mathcal{I}}\right)$.

We prove the result specifically for our problem in the appendix. Alternative conditions to characterise nondegenerate solutions of an optimal stopping problem are also discussed in Dayanik and Karatzas [9, Proposition 5.10]. In particular, they assume the conditions

$$
\lambda_{a_{\mathcal{I}}}=\limsup _{y \downarrow a_{\mathcal{I}}} g_{n}^{+}(S(y), x)<\infty \quad \text { and } \quad \lambda_{b_{\mathcal{I}}}=\limsup _{y \uparrow b_{\mathcal{I}}} \frac{g_{n}^{+}(S(y), x)}{S(y)}<\infty .
$$

From Proposition 3.2, denoting the smallest concave majorant of $g_{n}(\cdot, x)$ by $\bar{g}_{n}(\cdot, x)$, it follows that for $2 \leq n \leq N$,

$$
g_{n}(\theta, x)=\bar{g}_{n-1}\left(\theta, x+h^{n}\left(S^{-1}(\theta)\right)-h_{R}^{n}\right) .
$$

This implies that the value function at the $n$th step can be obtained by first solving for the value function at the $(n-1)$ th step.

\subsection{Piecewise exponential utility and drifting Brownian motion}

Having obtained a characterisation for the value function under partial liquidation, we apply the above methodology to the price process and preference function defined in Sects. 2.3 and 2.2, respectively. We limit our discussion to the case when $N=2$. The 
solutions for $N>2$ can be obtained through the same approach, but become rather unwieldy. Since our aim is to show that the investor may partially liquidate, we only need consider $N=2$ to show this.

We specialise to the case when the investor is selling or liquidating the asset itself; so we consider $h^{i}(y)=y$ for $i=1, \ldots, N$, with the common reference price $h_{R}=y_{R}$ for $i=1, \ldots, N$. We also interpret the reference price $y_{R}$ as the price at which the asset was purchased in the past. Before stating the main result, we first re-state the results obtained by Kyle et al. [24] and Henderson [14] for the case $N=1$, that is, when only block sales are allowed. [24] use a variational approach which is challenging due to the $S$-shaped utility function. Note that [24] do not include case (ii) below as they rule it out by an additional assumption on parameter values, perhaps because this assisted in the ease of their calculations.

Proposition 3.3 (Kyle et al. [24], Henderson [14]) Consider the optimal liquidation problem (2.1) with $N=1, h^{1}(y)=y$ and $h_{R}^{1}=y_{R}$. Suppose the price process $\left(Y_{t}\right)_{t \geq 0}$ is given by a Brownian motion with drift, $d Y_{t}=\mu d t+\sigma d W_{t}$ as in (2.4), and the utility function $U$ is the $S$-shaped piecewise exponential given by (2.2). Define $\eta=-2 \mu / \sigma^{2}$. The solution is given by the following four cases:

(i) If $\eta \leq 0$, the investor waits indefinitely and never liquidates.

(ii) If $0<\eta<\gamma_{1} \phi_{1} / \phi_{2}$, the investor always sells at a level $\bar{y}_{u}^{(1)}>y_{R}$ or above. The level $\bar{y}_{u}^{(1)}$ is given by

$$
\bar{y}_{u}^{(1)}=y_{R}-\frac{1}{\gamma_{1}} \ln \left(\frac{\phi_{1}+\phi_{2}}{\phi_{1}} \frac{2 \mu}{2 \mu-\gamma_{1} \sigma^{2}}\right) .
$$

(iii) If $0<\gamma_{1} \phi_{1} / \phi_{2} \leq \eta<\gamma_{2}$, the investor sells the first time the price process is equal to or exceeds the break-even level $y_{R}$.

(iv) If $\eta \geq \gamma_{2}$, the investor sells immediately at any price level.

We see from the above proposition for the block-sale problem that there are four cases depending upon the relative parameter values. Two cases are degenerate. If $\mu \geq 0$ as in case (i), the investor waits indefinitely regardless of price. Conversely, if the Sharpe ratio $\mu / \sigma^{2}$ is negative and sufficiently large compared to the risk seeking parameter $\gamma_{2}$, the investor sells immediately. The interesting situations arise between these extremes where we have two possibilities depending on where $\eta$ lies in the interval $0<\frac{\gamma_{1} \phi_{1}}{\phi_{2}}<\gamma_{2}$. In (iii) when the Sharpe ratio is relatively poor such that $\eta$ is in $\left[\frac{\gamma_{1} \phi_{1}}{\phi_{2}}, \gamma_{2}\right)$, the investor waits below the break-even level and liquidates at the break-even level $y_{R}$ itself. The width of the interval $\left[\frac{\gamma_{1} \phi_{1}}{\phi_{2}}, \gamma_{2}\right)$ reflects the strength of loss aversion in this model. If the investor is more loss averse, this interval is larger and a greater range of Sharpe ratios will fall into this case. In (ii) when the Sharpe ratio is better and $\eta \in\left(0, \frac{\gamma_{1} \phi_{1}}{\phi_{2}}\right)$, the investor waits beyond the break-even level and will not liquidate until the investment is in gains. Note that in (iii) and (iv), given our interpretation of the reference level as the price paid, liquidation will only occur at the break-even level itself. Effectively, the investor never holds the asset in these scenarios. Note also that the investor never waits to sell at a loss in this model.

Our interest in this paper is how the above generalises to partial liquidation. We can now state our extension to $N=2$ units. 
Proposition 3.4 Consider the optimal partial liquidation problem (2.1) with $N=2$, $h^{2}(y)=h^{1}(y)=y$ and $h_{R}^{2}=h_{R}^{1}=y_{R}$. Suppose the price process $\left(Y_{t}\right)_{t \geq 0}$ is given by a Brownian motion with drift, $d Y_{t}=\mu d t+\sigma d W_{t}$ as in (2.4), and the utility function $U$ is the $S$-shaped piecewise exponential given by (2.2). Define $\eta=-2 \mu / \sigma^{2}$. The solution consists of five cases depending on the relative parameter values:

(i) If $\eta \leq 0$, the investor waits indefinitely and never liquidates.

(ii) If $0<\eta<\gamma_{1} \phi_{1} / \phi_{2}$, the investor sells first at $\bar{y}_{u}^{(2)}$ and then at $\bar{y}_{u}^{(1)}$. (If $\bar{y}_{u}^{(2)} \geq \bar{y}_{u}^{(1)}$, the investor sells both assets at $\bar{y}_{u}^{(2)}$.)

(iii) The investor sells both units of asset at the price level $\bar{y}_{u}^{(2)}>y_{R}$ if

(a) $0<\eta / 2<\gamma_{2} \leq \eta$ and $\eta / 2<\gamma_{1} \phi_{1} / \phi_{2}$, or

(b) $0<\eta<\gamma_{2}$ and $\eta / 2<\gamma_{1} \phi_{1} / \phi_{2} \leq \eta$.

(iv) If $0<\eta / 2<\gamma_{2}$ and $\eta / 2 \geq \gamma_{1} \phi_{1} / \phi_{2}$, the investor sells both units of asset at $y_{R}$.

(v) If $0<\gamma_{2} \leq \eta / 2$, the investor sells immediately at all price levels.

The threshold $\bar{y}_{u}^{(2)}$ in (ii) and (iii) is given by

$$
\bar{y}_{u}^{(2)}=y_{R}-\frac{1}{2 \gamma_{1}} \ln \left(\frac{\phi_{1}+\phi_{2}}{\phi_{1}} \frac{\mu}{\mu-\gamma_{1} \sigma^{2}}\right) .
$$

Similarly to the case when only block sales are allowed (Proposition 3.3), the above proposition shows that under partial liquidation, the behaviour of the investor still depends on where the value of $\eta$ lies in comparison with scaled versions of the key quantities $\frac{\gamma_{1} \phi_{1}}{\phi_{2}}$ and $\gamma_{2}$. Thus again, decisions rely on the Sharpe ratio $\mu / \sigma^{2}$ of the asset together with the investor's risk aversion, risk seeking and loss aversion measures.

A first observation is that while we require $\frac{\gamma_{1} \phi_{1}}{\phi_{2}}<\gamma_{2}$ for loss aversion to hold, we may have either ordering of $\gamma_{2}$ and $\frac{2 \gamma_{1} \phi_{1}}{\phi_{2}}$, with the ordering $\frac{2 \gamma_{1} \phi_{1}}{\phi_{2}}<\gamma_{2}$ reflecting stronger loss aversion. We observe that the extreme cases where the investor waits indefinitely (see (i)) and sells immediately (see (v)) are still present when the asset can be partially sold. However, the Sharpe ratio of the asset needs to be worse compared to the block-sale model to put the investor in the 'sell immediately' case, since we now require $\eta \geq 2 \gamma_{2}$.

The situation we are most interested in occurs when $0<\eta<\frac{\gamma_{1} \phi_{1}}{\phi_{2}}$ (see (ii) above). This is the parameter regime when the Sharpe ratio, while negative, is better than all the other cases with $\eta>0$. In this parameter regime, the investor sells one unit of asset at the threshold $\bar{y}_{u}^{(2)}$ and the other unit of asset at the threshold $\bar{y}_{u}^{(1)}$. We have the following result.

Corollary 3.5 Let $0<\eta<\gamma_{1} \phi_{1} / \phi_{2}$. If the parameters are such that

$$
\frac{\phi_{2}}{\phi_{1}} \frac{2 \eta}{\gamma_{1}}\left(1+\frac{\eta}{2 \gamma_{1}}\right)<1,
$$

then we have $\bar{y}_{u}^{(2)}<\bar{y}_{u}^{(1)}$ and the asset will be sold at two distinct thresholds. Otherwise, $\bar{y}_{u}^{(2)} \geq \bar{y}_{u}^{(1)}$ and both units of asset are sold together at the threshold $\bar{y}_{u}^{(2)}$. 


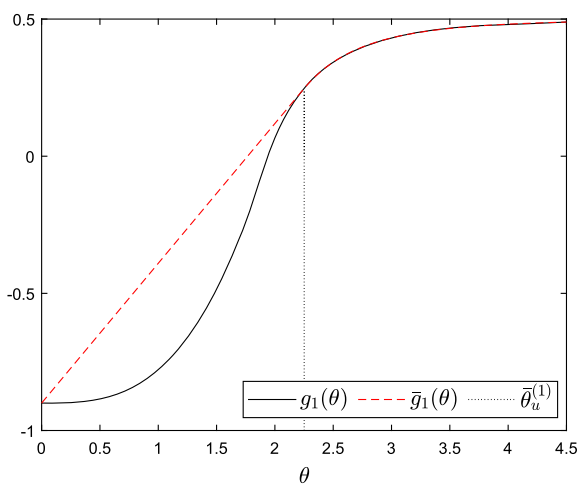

(a) Case (ii). $\quad g_{1}(\theta)$ vs. $\bar{g}_{1}(\theta)$

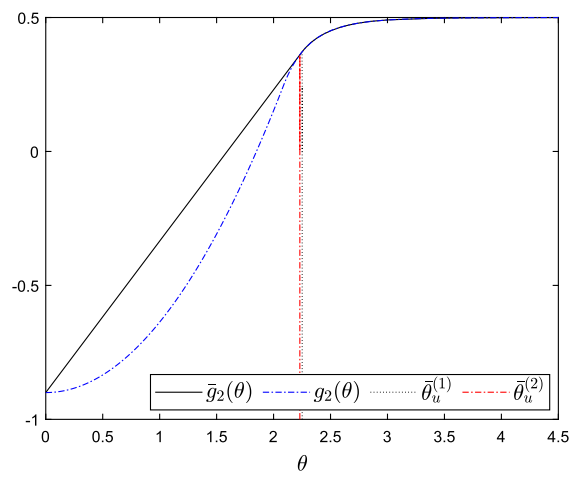

(c) Case (ii). $g_{2}(\theta)$ vs. $\bar{g}_{2}(\theta) \cdot\left(\bar{\theta}_{u}^{(1)}=2.251\right.$, $\bar{\theta}_{u}^{(2)}=2.230, \bar{y}_{u}^{(1)}=1.227$ and $\left.\bar{y}_{u}^{(2)}=1.213\right)$

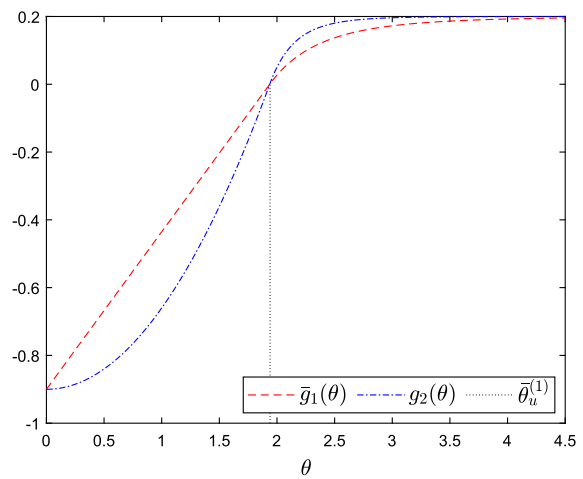

(e) Case (ii). $\quad \bar{g}_{1}(\theta)$ vs. $g_{2}(\theta)$.

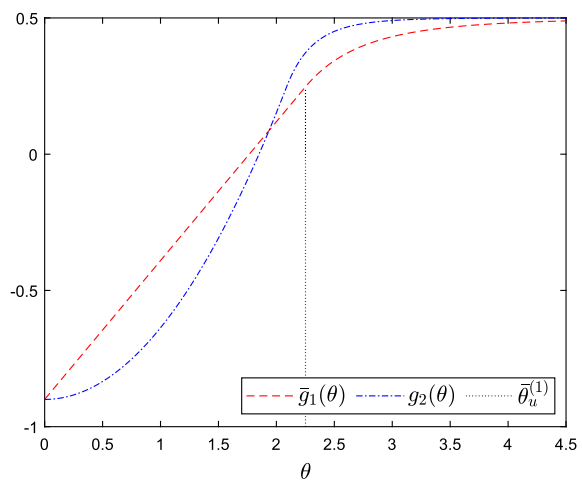

(b) Case (ii). $\quad \bar{g}_{1}(\theta)$ vs. $g_{2}(\theta)$.

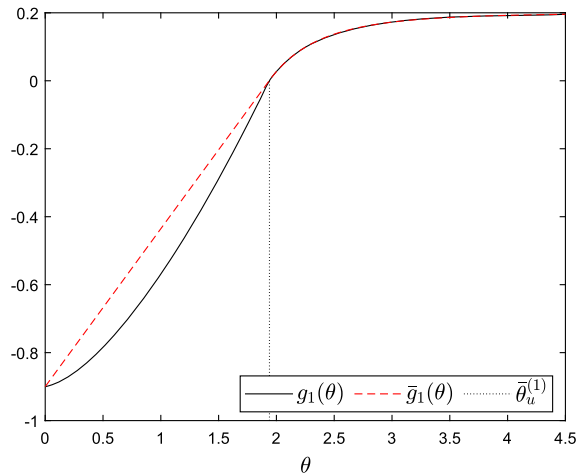

(d) Case (ii). $\quad g_{1}(\theta)$ vs. $\bar{g}_{1}(\theta)$.

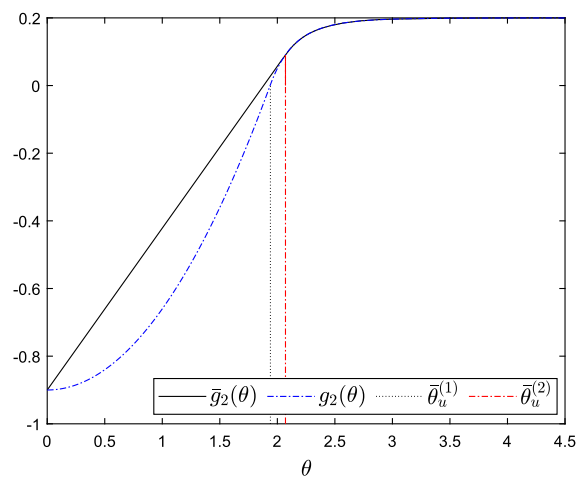

(f) Case (ii). $g_{2}(\theta)$ vs. $\bar{g}_{2}(\theta) \cdot\left(\bar{\theta}_{u}^{(1)}=1.940\right.$, $\bar{\theta}_{u}^{(2)}=2.071, \bar{y}_{u}^{(1)}=1.002$ and $\left.\bar{y}_{u}^{(2)}=1.101\right)$

Fig. 2 Optimal liquidation of two units of asset under the model of Proposition 3.4, case (ii). Panels (a)-(c) use the parameters $\phi_{1}=0.5, \phi_{2}=0.9, \gamma_{1}=3, \gamma_{2}=2$ and $\eta=0.66$. Condition (3.9) is satisfied, giving $\bar{y}_{u}^{(2)}=1.213<\bar{y}_{u}^{(1)}=1.227$. Panels (d)-(f) use the parameters $\phi_{1}=0.2, \phi_{2}=0.9, \gamma_{1}=3, \gamma_{2}=1$ and $\eta=0.66$. Condition (3.9) is not satisfied for this choice; hence both units are sold at $\bar{y}_{u}^{(2)}=1.101$. All panels use a reference level (per unit) of $y_{R}=1$. Set $x=0$ 


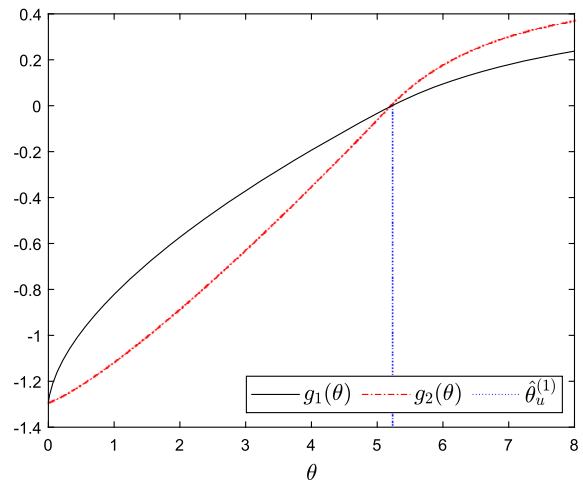

(a) Case (iii)(a). $g_{1}(\theta)$ vs. $g_{2}(\theta)$.

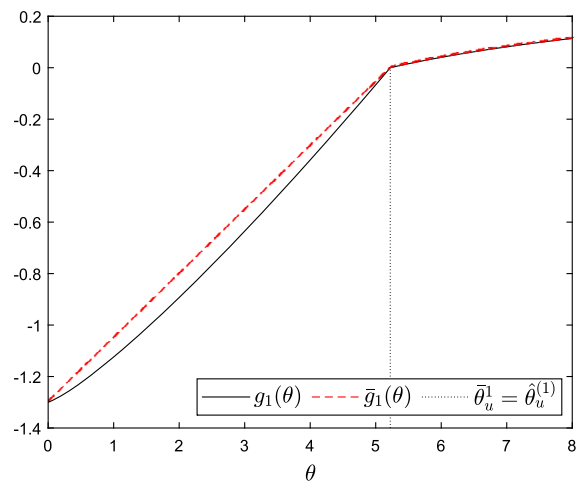

(c) Case (iv). $\quad g_{1}(\theta)$ vs. $\bar{g}_{1}(\theta)$.

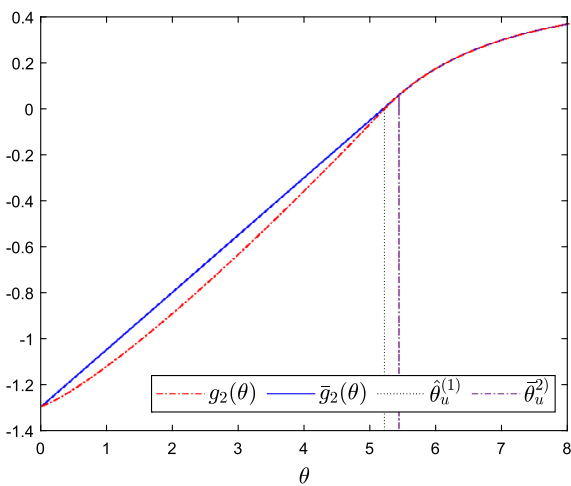

(b) Case (iii)(a). $g_{2}(\theta)$ vs. $\bar{g}_{2}(\theta) \cdot\left(\bar{\theta}_{u}^{(2)}=5.418\right.$ and $\left.\bar{y}_{u}^{(2)}=1.022\right)$

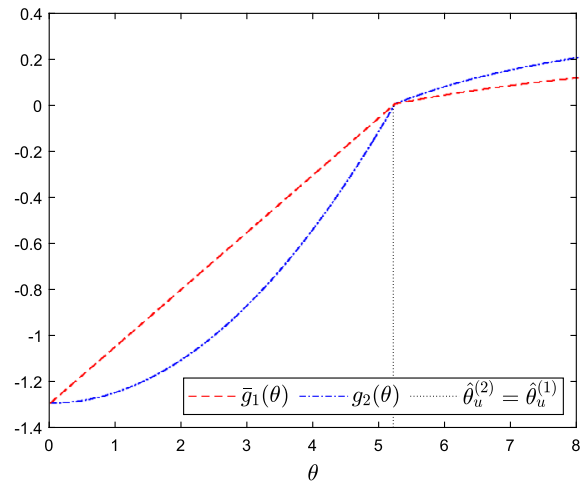

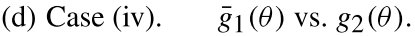

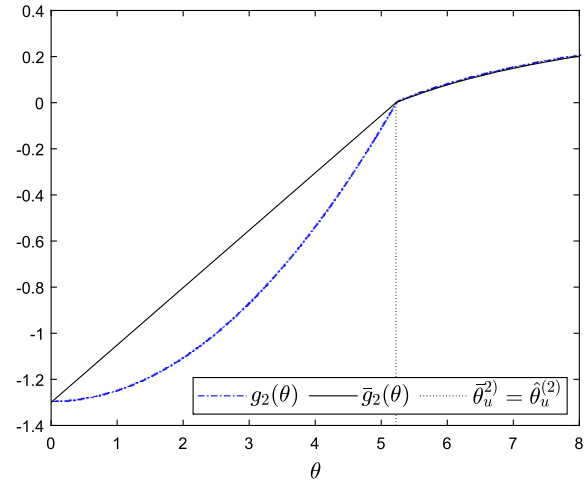

(e) Case (iv). $g_{2}(\theta)$ vs. $\bar{g}_{2}(\theta) \cdot\left(\bar{\theta}_{u}^{(1)}=\bar{\theta}_{u}^{(2)}=\right.$ 5.22, $\left.\bar{y}_{u}^{(1)}=\bar{y}_{u}^{(2)}=1\right)$

Fig. 3 Optimal liquidation of two units of asset under the model of Proposition 3.4, cases (iii)(a) and (iv). Panels (a) and (b) illustrate case (iii)(a) with $\phi_{1}=0.5, \phi_{2}=1.3, \gamma_{1}=2.5, \gamma_{2}=1$ and $\eta=1.65$. Both units are sold at $\bar{y}_{u}^{(2)}=1.022>y_{R}$. Panels (c)-(e) illustrate case (iv) with $\phi_{1}=0.5, \phi_{2}=1.3, \gamma_{1}=1$, $\gamma_{2}=2$ and $\eta=1.64$. Both units are sold at $\bar{y}_{u}^{(1)}=\bar{y}_{u}^{(2)}=1=y_{R}$. All panels use a reference level (per unit) of $y_{R}=1$. Set $x=0$ 
The condition in (3.9) splits the relevant region of parameters into two sub-regions. If the parameters are such that $0<\eta<\gamma_{1}\left(\sqrt{1 / 2+\phi_{1} / \phi_{2}}-1\right)$, we have $\bar{y}_{u}^{(2)}>\bar{y}_{u}^{(1)}$ and the asset is sold at two distinct thresholds. This sub-region of parameters is the slightly better one, where the value of $\eta$ is small and positive and hence the expected return is negative but small in magnitude. This is the regime where the investor is willing to wait and sell at different thresholds, as the risk of waiting is not too great to bear relative to returns. Otherwise, $\gamma_{1}\left(\sqrt{1 / 2+\phi_{1} / \phi_{2}}-1\right)<\eta<\gamma_{1} \phi_{1} / \phi_{2}$ and then the units of asset are sold together at the threshold $\bar{y}_{u}^{(2)}$. In this sub-region, the value of $\eta$ is a little larger and hence the expected return is worse. Hence here, the investor would prefer to sell both units of asset at once as the risk of waiting longer to reach a higher threshold is too great relative to the weaker returns. Finally, we can also remark that for stronger loss aversion, the region of parameters where the investor sells at two distinct thresholds shrinks.

Figure 2 illustrates the results from case (ii). All panels use a reference level (per unit) of $y_{R}=1$. Panels (a)-(c) use the parameters $\phi_{1}=0.5, \phi_{2}=0.9, \gamma_{1}=3, \gamma_{2}=2$ and $\eta=0.66$. Condition (3.9) is satisfied, giving $\bar{y}_{u}^{(2)}=1.213<\bar{y}_{u}^{(1)}=1.227$. Panels (d)-(f) use the parameters $\phi_{1}=0.2, \phi_{2}=0.9, \gamma_{1}=3, \gamma_{2}=1$ and $\eta=0.66$. Condition (3.9) is not satisfied for this choice; hence both units are sold at $\bar{y}_{u}^{(2)}=1.101$.

The remaining cases (iii) and (iv) involve selling both units of asset simultaneously, either at a gain threshold $\bar{y}_{u}^{(2)}$ (case (iii)) or at the break-even level $y_{R}$ (case (iv)). Figure 3 illustrates the results from cases (iii)(a) and (iv). Again, all panels use a reference level (per unit) of $y_{R}=1$. Panels (a) and (b) illustrate case (iii)(a). The parameters are $\phi_{1}=0.5, \phi_{2}=1.3, \gamma_{1}=2.5, \gamma_{2}=1$ and $\eta=1.65$. We see both units are sold at $\bar{y}_{u}^{(2)}=1.022>y_{R}$. Panels (c)-(e) illustrate case (iv). The parameters are $\phi_{1}=0.5, \phi_{2}=1.3, \gamma_{1}=1, \gamma_{2}=2$ and $\eta=1.64$. Both units are sold at $\bar{y}_{u}^{(1)}=\bar{y}_{u}^{(2)}=1=y_{R}$.

To demonstrate the difference between the solutions in the block-sale and partialsale models, consider an example where $\eta \in\left(\frac{\gamma_{1} \phi_{1}}{\phi_{2}}, \gamma_{2}\right)$. If only block sales are allowed, Proposition 3.3 (iii) says that the investor sells the first time the price process is equal to or exceeds the break-even level $y_{R}$ and hence, under our assumptions, effectively never enters the problem in this parameter regime. Once we allow partial sales, there are two possibilities. The investor may still sell at $y_{R}$ (and thus effectively never enter), but only if the loss aversion is relatively strong in that we have $\frac{2 \gamma_{1} \phi_{1}}{\phi_{2}}<\eta<\gamma_{2}$ (see Proposition 3.4, case (iv)). The other possibility is that the investor sells both units of asset at a gain, at the threshold $\bar{y}_{u}^{(2)}$; see Proposition 3.4, case (iii)(b).

\section{Discussion and conclusions}

Researchers have studied multiple optimal stopping problems under standard concave utility functions in other settings. For example, Grasselli and Henderson [12], Leung and Sircar [25] and Henderson and Hobson [15] consider the exercise of American options under concave utilities and demonstrate that the solution involves exercising 
a tranche of (identical) options over different asset price thresholds. Intuitively, a riskaverse investor wants to spread the risk of continuing to hold the options by exercising them separately. Similarly, intuition would tell us that an investor who is risk-seeking with convex utility would prefer to engage in a block sale. What might we expect from an $S$-shaped reference-dependent utility? Since there are concave and convex parts to the utility, we could reasonably expect that either might be dominant, depending on parameters. Somewhat surprisingly, Henderson [14] showed that under a Tversky and Kahneman [33] $S$-shaped function and exponential Brownian motion, the investor's optimal strategy, when not degenerate, always involved selling both units of asset together. In this paper, we demonstrate that with piecewise exponential $S$-shaped functions and drifting Brownian price dynamics, it is possible to obtain a situation where the investor chooses to sell her asset gradually rather than in a block.

Our results suggest that it would be worthwhile for experimental tests of optimal stopping under reference-dependent preferences to extend their focus to consider the question of how individuals sell a divisible quantity of asset. Potential further theoretical work may examine the additional feature of an exogenous end of game, where the asset is liquidated upon arrival of the first jump of a Poisson process (see Kyle et al. [24], Barberis and Xiong [4] for examples). This would improve the applicability of the model, as it would mean that an investor would sometimes sell, rather than hold, when the Sharpe ratio is positive. While injecting realism, this addition would be at the expense of tractability of the solution method, and for this reason, we do not pursue it here.

Acknowledgements We thank participants at the QMF conference (Sydney, December 2018), the Bachelier World Congress (Dublin, July 2018) and the 11th European Summer School in Financial Mathematics (Paris, August 2018).

Publisher's Note Springer Nature remains neutral with regard to jurisdictional claims in published maps and institutional affiliations.

Open Access This article is licensed under a Creative Commons Attribution 4.0 International License, which permits use, sharing, adaptation, distribution and reproduction in any medium or format, as long as you give appropriate credit to the original author(s) and the source, provide a link to the Creative Commons licence, and indicate if changes were made. The images or other third party material in this article are included in the article's Creative Commons licence, unless indicated otherwise in a credit line to the material. If material is not included in the article's Creative Commons licence and your intended use is not permitted by statutory regulation or exceeds the permitted use, you will need to obtain permission directly from the copyright holder. To view a copy of this licence, visit http://creativecommons.org/licenses/by/4.0/.

\section{Appendix A}

Lemma A.1 Let $\tau_{n}$ be an $\mathbb{F}$-stopping time and $f$ an increasing continuous function satisfying $f(0)=0$. Then the family $\Gamma=\left\{Z^{\alpha}: \alpha \in \mathcal{I}\right\}$ of $\mathcal{F}_{\tau_{n}}$-measurable random variables defined in the proof of Proposition 3.1 has the lattice property.

Proof Let $\alpha, \alpha^{\prime} \in \mathcal{I}$ so that $\alpha=\left(\xi_{n-1}, \ldots, \xi_{1}\right)$ and $\alpha^{\prime}=\left(\xi_{n-1}^{\prime}, \ldots, \xi_{1}^{\prime}\right)$ satisfy

$$
\tau_{n} \leq \xi_{n-1} \leq \cdots \leq \xi_{1} \quad \text { and } \quad \tau_{n} \leq \xi_{n-1}^{\prime} \leq \cdots \leq \xi_{1}^{\prime} .
$$


Recall that

$$
Z^{\alpha}=\mathbb{E}\left[f\left(Y_{\tau_{n}}+\sum_{i=1}^{n-1} Y_{\xi_{i}}\right) \mid \mathcal{F}_{\tau_{n}}\right] \quad \text { and } \quad Z^{\alpha^{\prime}}=\mathbb{E}\left[f\left(Y_{\tau_{n}}+\sum_{i=1}^{n-1} Y_{\xi_{i}^{\prime}}\right) \mid \mathcal{F}_{\tau_{n}}\right]
$$

Consider $\bar{\alpha}=\left(\bar{\xi}_{n-1}, \ldots, \bar{\xi}_{1}\right) \in \mathcal{I}$ defined by ${ }^{2}$

$$
\bar{\xi}_{i}=\xi_{i} \mathbb{I}_{\left\{Z^{\alpha} \geq Z^{\alpha^{\prime}}\right\}}+\xi_{i}^{\prime} \mathbb{I}_{\left\{Z^{\alpha}<Z^{\alpha^{\prime}}\right\}} .
$$

Then it follows that

$$
\begin{aligned}
Z^{\bar{\alpha}} & =\mathbb{E}\left[f\left(Y_{\tau_{n}}+\sum_{i=1}^{n-1} Y_{\xi_{i}}\right) \mathbb{I}_{\left\{Z^{\alpha} \geq Z^{\left.\alpha^{\prime}\right\}}\right.}+f\left(Y_{\tau_{n}}+\sum_{i=1}^{n-1} Y_{\xi_{i}^{\prime}}\right) \mathbb{I}_{\left\{Z^{\alpha}<Z^{\left.\alpha^{\prime}\right\}}\right.} \mid \mathcal{F}_{\tau_{n}}\right] \\
& =Z^{\alpha} \mathbb{I}_{\left\{Z^{\alpha} \geq Z^{\left.\alpha^{\prime}\right\}}\right.}+Z^{\alpha^{\prime}} \mathbb{I}_{\left\{Z^{\alpha}<Z^{\left.\alpha^{\prime}\right\}}\right.}=\max \left(Z^{\alpha}, Z^{\alpha^{\prime}}\right) .
\end{aligned}
$$

Proof of Proposition 3.2 Both results are proved for $n=1$ as the same arguments can be easily extended for general $n$.

(i) Fix $Y_{0}=y$ and consider a sequence $b_{n} \uparrow b_{\mathcal{I}}$ with $y \leq b_{n}$. Furthermore, consider the local martingale $\Theta=S(Y)$ and let $\tau_{n}=\tau_{\left(a_{\mathcal{I}}, b_{n}\right)}^{Y}=\tau_{\left(-\infty, S\left(b_{n}\right)\right)}^{\Theta}$. As $U$ is increasing in $y$, we have $V_{1}(y, x) \leq \lim _{n \rightarrow \infty} U\left(h^{1}\left(b_{n}\right)-h_{R}^{1}+x\right)$ for all $y \in \mathcal{I}$.

Let $\theta=S(y)$ and take $a \in \mathbb{R}$ such that $-\infty<a \leq \theta$. Since the local martingale $\Theta$ is in natural scale, $\Theta$ starting at $\theta$ leaves the interval $\left(S(a), S\left(b_{n}\right)\right)$ at $S\left(b_{n}\right)$ with probability $\frac{S(\theta)-S(a)}{S\left(b_{n}\right)-S(a)}$. By taking $a \downarrow-\infty$, it follows that $\mathbb{P}\left[\tau_{\left(-\infty, S\left(b_{n}\right)\right)}^{\Theta}<\infty\right]=1$. This in turn implies that

$$
\begin{aligned}
V_{1}(y, x) & =\sup _{\tau} \mathbb{E}_{y}\left[U\left(h^{1}\left(Y_{\tau}\right)-h_{R}^{1}+x\right)\right] \\
& \geq \mathbb{E}_{y}\left[U\left(h^{1}\left(Y_{\tau_{n}}\right)-h_{R}^{1}+x\right)\right] \\
& =U\left(h^{1}\left(b_{n}\right)-h_{R}^{1}+x\right),
\end{aligned}
$$

where the last equality follows from the fact that $\Theta$ exits from the interval $\left(-\infty, S\left(b_{n}\right)\right)$ at $S\left(b_{n}\right)$ almost surely, as shown above. But this is true for all $n \in \mathbb{N}$ and hence by taking limits in $n$, we have $V_{1}(y, x) \geq U\left(h^{1}\left(b_{\mathcal{I}}\right)-h_{R}^{1}+x\right)$, giving the result.

(ii) Hold $x$ constant. By definition, we have

$$
V_{1}(y, x)=\sup _{\tau} \mathbb{E}\left[U\left(h^{1}\left(Y_{\tau}\right)-h_{R}^{1}+x\right) \mid Y_{0}=y\right]=\sup _{\tau} \mathbb{E}\left[g_{1}\left(\Theta_{\tau}, x\right) \mid \Theta_{0}=\theta\right],
$$

where $g_{1}(\theta, x)=U\left(S^{-1}(\theta)-y_{R}+x\right)$ as in (3.5). Since $U(\cdot)$ is increasing, also $g_{1}(\cdot, x)$ is increasing and we let $\tilde{g}_{1}(\cdot, x)$ be any increasing, concave majorant of $g_{1}(\cdot, x)$. But for every stopping rule $\tau$,

$$
\mathbb{E}\left[g_{1}\left(\Theta_{\tau}, x\right) \mid \Theta_{0}=\theta\right] \leq \mathbb{E}\left[\tilde{g}_{1}\left(\Theta_{\tau}, x\right) \mid \Theta_{0}=\theta\right] \leq \tilde{g}_{1}\left(\mathbb{E}\left[\Theta_{\tau} \mid \Theta_{0}=\theta\right], x\right)
$$

\footnotetext{
${ }^{2}$ The fact that each $\bar{\xi}_{i}$ is a stopping time follows from the fact that $Z^{\alpha}$ and $Z^{\alpha^{\prime}}$ are $\mathcal{F}_{\tau_{n}}$-measurable.
} 
where we use the fact that $\tilde{g}_{1}(\cdot, x)$ is a concave majorant of $g_{1}(\cdot, x)$ and Jensen's inequality. Finally, because $\Theta \geq S\left(a_{\mathcal{I}}\right)>-\infty$ is a local martingale bounded below and hence a supermartingale, we obtain

$$
\tilde{g}_{1}\left(\mathbb{E}\left[\Theta_{\tau} \mid \Theta_{0}=\theta\right], x\right) \leq \tilde{g}_{1}(\theta, x) .
$$

The arbitrariness of the stopping time $\tau$ gives $V_{1}(y, x) \leq \bar{g}_{1}(\theta, x)$, where $\bar{g}_{1}(\cdot, x)$ is the smallest concave majorant of $g_{1}(\cdot, x)$. Note that because $U(\cdot)=g_{1}(\cdot, x)$ is increasing, so is its smallest concave majorant $\bar{g}_{1}(\cdot, x)$. Thus we have shown that we cannot do better than $\bar{g}_{1}(\cdot, x)$ when allowing general stopping rules. It remains to show that there is a stopping rule which attains this bound.

Suppose first that $\lim \sup _{\psi \uparrow S\left(b_{\mathcal{I}}\right)} \frac{g_{1}(\psi, x)}{\psi-S\left(a_{\mathcal{I}}\right)}=\infty$, implying that $\bar{g}_{1}(\theta, x)=\infty$ for $\theta \in\left(S\left(a_{\mathcal{I}}\right), S\left(b_{\mathcal{I}}\right)\right)$. Then there exists a sequence $b_{n} \uparrow b_{\mathcal{I}}$ such that $\frac{g_{1}\left(S\left(b_{n}\right), x\right)}{S\left(b_{n}\right)-S\left(a_{\mathcal{I}}\right)} \uparrow \infty$ as $n \uparrow \infty$. Given $\theta \in\left(S\left(a_{\mathcal{I}}\right), S\left(b_{\mathcal{I}}\right)\right)$ and $S\left(a_{\mathcal{I}}\right)<\hat{\phi}<\theta$, define the sequence of stopping rules $\tau_{n}^{*}=\tau_{\left(\hat{\phi}, S\left(b_{n}\right)\right)}^{\Theta}=\tau_{\left(S^{-1}(\hat{\phi}), b_{n}\right)}^{Y}$ which converges almost surely to $\tau^{*}=\tau_{\left(\hat{\phi}, S\left(b_{\mathcal{I}}\right)\right)}^{\Theta}=\tau_{\left(S^{-1}(\hat{\phi}), b_{\mathcal{I}}\right)}^{Y}$. (This follows easily from the definition of the stopping times). Then by using the transformation in (3.5) and the fact that the process $\Theta$ is in natural scale, we have

$$
\begin{aligned}
& \mathbb{E}\left[U\left(h^{1}\left(Y_{\tau_{n}^{*}}\right)-h_{R}^{1}+x\right) \mid Y_{0}=S^{-1}(\theta)\right] \\
& =\mathbb{E}\left[g_{1}\left(\Theta_{\tau_{n}^{*}}, x\right) \mid \Theta_{0}=\theta\right] \\
& =g_{1}(\hat{\phi}, x) \frac{S\left(b_{n}\right)-\theta}{S\left(b_{n}\right)-\hat{\phi}}+g_{1}\left(S\left(b_{n}\right), x\right) \frac{\theta-\hat{\phi}}{S\left(b_{n}\right)-\hat{\phi}} .
\end{aligned}
$$

Taking limits in $n$, it hence follows that for some constant $K>0$,

$$
\begin{aligned}
& \mathbb{E}\left[U\left(h^{1}\left(Y_{\tau^{*}}\right)-h_{R}^{1}+x\right) \mid Y_{0}=S^{-1}(\theta)\right] \\
& =\lim _{n \rightarrow \infty}\left(g_{1}(\hat{\phi}, x) \frac{S\left(b_{n}\right)-\theta}{S\left(b_{n}\right)-\hat{\phi}}+g_{1}\left(S\left(b_{n}\right), x\right) \frac{\theta-\hat{\phi}}{S\left(b_{n}\right)-\hat{\phi}}\right) \\
& \geq K+(\theta-\hat{\phi}) \lim _{n \rightarrow \infty} \frac{g_{1}\left(S\left(b_{n}\right), x\right)}{S\left(b_{n}\right)-S\left(a_{\mathcal{I}}\right)} \\
& =\infty,
\end{aligned}
$$

and hence $\tau^{*}$ attains the bound.

Now suppose that $\lim \sup _{\psi \uparrow S\left(b_{\mathcal{I}}\right)} \frac{g_{1}(\psi, x)}{\psi-S\left(a_{\mathcal{I}}\right)}<\infty$. In this case, $\bar{g}_{1}(\cdot, x)$ is a finitevalued function. Fix $\theta$ and let $\Upsilon=\left\{v: \bar{g}_{1}(v, x)=g_{1}(v, x)\right\}$. Suppose $\theta \in \Upsilon$. Then with $\tau=0$, by again applying the transformation in (3.5), we are done because

$$
\mathbb{E}\left[U\left(h^{1}\left(Y_{0}\right)-h_{R}^{1}+x\right) \mid Y_{0}=y\right]=g_{1}(\theta, x)=\bar{g}_{1}(\theta, x) .
$$

Otherwise, define $\phi^{*}=\sup \{\xi<\theta: \xi \in \Upsilon\}, \psi^{*}=\inf \{\xi>\theta: \theta \in \Upsilon\}$ with $\phi^{*}=S\left(a_{\mathcal{I}}\right)$ if $\{\xi<\theta: \theta \in \Upsilon\}=\emptyset$ and $\psi^{*}=S\left(b_{\mathcal{I}}\right)$ if $\{\xi>\theta: \theta \in \Upsilon\}=\emptyset$. 
Suppose $\psi^{*}<\infty$. Then $\theta \mapsto \bar{g}_{1}(\theta, x)$ is linear on the interval $\left(\phi^{*}, \psi^{*}\right)$ and $\left(\Theta_{t \wedge \tau_{\left(\phi^{*}, \psi^{*}\right)}}\right)$ is a martingale. Letting $\tau^{*}=\tau_{\left(\phi^{*}, \psi^{*}\right)}^{\Theta}=\tau_{\left(S^{-1}\left(\phi^{*}\right), S^{-1}\left(\psi^{*}\right)\right)}^{Y}$, we have

$$
\begin{aligned}
\mathbb{E}\left[U\left(h^{1}\left(Y_{\tau^{*}}\right)-h_{R}^{1}+x\right) \mid Y_{0}=y\right] & =\mathbb{E}\left[g_{1}\left(\Theta_{\tau_{\left(\phi^{*}, \psi^{*}\right)}}, x\right)\right] \\
& =g_{1}\left(\phi^{*}, x\right) \frac{\psi^{*}-\theta}{\psi^{*}-\phi^{*}}+g_{1}\left(\psi^{*}, x\right) \frac{\theta-\phi^{*}}{\psi^{*}-\phi^{*}} \\
& =\bar{g}_{1}(\theta, x) .
\end{aligned}
$$

On the other hand, if $\psi^{*}=\infty$, then $\bar{g}_{1}(\cdot, x)$, the smallest concave majorant of $g_{1}(\cdot, x)$, is constant over $\left(\phi^{*}, \infty\right)$. Consider the stopping time $\tau^{*}=\tau_{\left(\phi^{*}, \infty\right)}^{\Theta}=\tau_{\left(S^{-1}\left(\phi^{*}\right), \infty\right)}^{Y}$ and an increasing sequence $\left(\theta_{n}\right)$ with $\theta_{1}>\theta$ and $\theta_{n} \uparrow \infty$. Since both $g_{1}(\cdot, x)$ and $\bar{g}_{1}(\cdot, x)$ are finite-valued functions and $\psi^{*}=\infty$, we have

$$
\begin{aligned}
\mathbb{E}\left[U\left(h^{1}\left(Y_{\tau^{*}}\right)-h_{R}^{1}+x\right) \mid Y_{0}=y\right] & =\lim _{n \rightarrow \infty}\left(\bar{g}_{1}\left(\phi^{*}, x\right) \frac{\theta_{n}-\theta}{\theta_{n}-\phi^{*}}+\bar{g}_{1}\left(\theta_{n}, x\right) \frac{\theta-\phi^{*}}{\theta_{n}-\phi^{*}}\right) \\
& =\lim _{n \rightarrow \infty} \bar{g}_{1}(\phi, x) \frac{\theta_{n}-\phi^{*}}{\theta_{n}-\phi^{*}} \\
& =\bar{g}_{1}(\phi, x)=\bar{g}_{1}(\theta, x) .
\end{aligned}
$$

Proof of Proposition 3.3 Consider first the case when $\mu>0$ (i.e., $\eta<0$ ). The state space $\mathcal{I}=(-\infty, \infty)$ of $Y$ has natural endpoints and $S(\mathcal{I})=(-\infty, 0)$. Then by Proposition 3.2, $V_{1}(y, x)=U\left(x+b_{\mathcal{I}}-h_{R}^{1}\right)=\infty$. This gives case (i).

Now consider the case when $\mu<0$ (i.e., $\eta>0)$. Then we have $S(\mathcal{I})=(0, \infty)$, $S\left(y_{R}\right)=\exp \left(-2 \mu y_{R} / \sigma^{2}\right)$ and hence $S^{-1}(\theta)=-\left(\sigma^{2} /(2 \mu)\right) \ln \theta$ for $\theta \in(0, \infty)$. Fix $x$. Then the stopping problem translates into finding the function $\bar{g}_{1}(\cdot, x)$, the smallest concave majorant of the function $g_{1}(\cdot, x)$. Using the definition of $U(\cdot)$ in (2.2) and the fact that $g_{1}(\theta, x)$ is given by

$$
g_{1}(\theta, x)=U\left(S^{-1}(\theta)-y_{R}+x\right)
$$

as in (3.5), we have

$$
g_{1}(\theta, x)= \begin{cases}\phi_{1}\left(1-\exp \left(-\gamma_{1}\left(y_{R}-x\right)\right) \theta^{\gamma_{1} \frac{\sigma^{2}}{2 \mu}}\right) & \text { for } \theta \geq \exp \left(\eta\left(y_{R}-x\right)\right), \\ \phi_{2}\left(\exp \left(\gamma_{2}\left(y_{R}-x\right)\right) \theta^{-\gamma_{2} \frac{\sigma^{2}}{2 \mu}}-1\right) & \text { for } \theta<\exp \left(\eta\left(y_{R}-x\right)\right) .\end{cases}
$$

By differentiating $g_{1}(\cdot, x)$ and observing the behaviour of $g_{1}^{\prime}(\theta, x)$ at $\theta=0$, it follows that for $\gamma_{2} \leq \eta, g_{1}(\cdot, x)$ is concave on both $(0, \hat{\theta})$ and $(\hat{\theta}, \infty)$, where $\hat{\theta}=\exp \left(\eta \gamma_{R}\right)$. Furthermore, since $\lim _{\theta \uparrow \hat{\theta}} g_{1}^{\prime}(\theta, 0)<\lim _{\theta \downarrow \hat{\theta}} g_{1}^{\prime}(\theta, 0)$, it follows that $g_{1}(\cdot, x)$ is concave over $(0, \infty)$. This implies that $\bar{g}_{1}(\cdot, x)=g_{1}(\cdot, x)$ and hence the stopping region coincides with the whole state space $\mathcal{I}$. This gives case (iv).

When $\gamma_{2}>\eta$, it follows that $\theta \mapsto g_{1}(\theta, x)$ is convex for $\theta<\exp \left(\eta y_{R}\right)$ and concave otherwise. Thus the smallest concave majorant $\bar{g}_{1}(\cdot, x)$ of $g_{1}(\cdot, x)$ is obtained by considering the chord from the point $\left(0,-\phi_{2}\right)$ to a point $\left(\bar{\theta}_{u}, g_{1}\left(\bar{\theta}_{u}, x\right)\right)$ at which 
the slope of the chord is equal to the slope of $g_{1}(\cdot, x)$ for $\theta \geq S\left(y_{R}\right)$. Since we are after $V_{1}(y, 0)$, setting $x=0$ gives

$$
\bar{y}_{u}^{(1)}=y_{R}-\frac{1}{\gamma_{1}} \ln \left(\frac{\phi_{1}+\phi_{2}}{\phi_{1}} \frac{2 \mu}{2 \mu-\gamma_{1} \sigma^{2}}\right) .
$$

Since $\bar{y}_{u}^{(1)} \geq y_{R}$, it follows from (A.1) that $\bar{y}_{u}^{(1)}>y_{R}$ for $0<\eta<\left(\phi_{1} / \phi_{2}\right) \gamma_{1}$ (case (ii)) and $\bar{y}_{u}^{(1)}=y_{R}$ otherwise (case (iii)).

Proof of Proposition 3.4 Suppose $\mu>0$ (i.e., $\eta<0$ ). Similar arguments as for case (i) in Proposition 3.3 show that under this assumption, the investor will wait indefinitely and never liquidate their position (case (i)).

Henceforth we assume that $\eta>0$ so that $\mu<0$. As described in Sect. 3.1, the solution of this problem can be found by first characterising $V_{1}(y, x)$ through the transformed reward function $g_{1}(\cdot, x)$ and its smallest concave majorant $\bar{g}_{1}(\cdot, x)$. We then use the relation

$$
g_{n}(\theta, x)=V_{n-1}\left(S^{-1}(\theta), x+h^{n}\left(S^{-1}(\theta)-h_{R}^{n}\right)\right)=\bar{g}_{n-1}\left(\theta, x+S^{-1}(\theta)-y_{R}\right)
$$

to characterise $V_{2}(y, x)$ and the corresponding optimal stopping region. Given that $N=2$, setting $x=0$ gives $V(y)=V_{2}(y, 0)$ and we are done. Given that the problem characterising $V_{1}(y, x)$ is identical to that described in Proposition 3.3, we know that

$$
g_{1}(\theta, x)= \begin{cases}\phi_{1}\left(1-\exp \left(-\gamma_{1}\left(y_{R}-x\right)\right) \theta^{\gamma_{1} \frac{\sigma^{2}}{2 \mu}}\right) & \text { for } \theta \geq \exp \left(\eta\left(y_{R}-x\right)\right), \\ \phi_{2}\left(\exp \left(\gamma_{2}\left(y_{R}-x\right)\right) \theta^{-\gamma_{2} \frac{\sigma^{2}}{2 \mu}}-1\right) & \text { for } \theta<\exp \left(\eta\left(y_{R}-x\right)\right) .\end{cases}
$$

Suppose $\eta \geq \gamma_{2}$. From the proof of Proposition 3.3, case (iv), we know that this assumption implies $\bar{g}_{1}(\theta, x)=g_{1}(\theta, x)$. By applying (A.2), we have

$$
g_{2}(\theta, x)=\bar{g}_{1}\left(\theta, x+S^{-1}(\theta)-y_{R}\right)=g_{1}\left(\theta, x+S^{-1}(\theta)-y_{R}\right),
$$

and using $S^{-1}(\theta)=-\left(\sigma^{2} /(2 \mu)\right) \ln \theta$ gives

$$
g_{2}(\theta, x)= \begin{cases}\phi_{1}\left(1-\exp \left(-\gamma_{1}\left(2 y_{R}-x\right)\right) \theta^{\gamma_{1} \frac{\sigma^{2}}{\mu}}\right) & \text { for } \theta \geq \hat{\theta}^{(2)}(x), \\ \phi_{2}\left(\exp \left(\gamma_{2}\left(2 y_{R}-x\right)\right) \theta^{-\gamma_{2} \frac{\sigma^{2}}{\mu}}-1\right) & \text { for } \theta<\hat{\theta}^{(2)}(x),\end{cases}
$$

where $\hat{\theta}^{(2)}(x)=\exp \left(\frac{\eta}{2}\left(2 y_{R}-x\right)\right)$. By differentiating $g_{2}(\cdot, x)$ with respect to $\theta$ and setting $x=0$, we find that

(a-i) $g_{2}^{\prime}(\theta, 0) \downarrow 0$ as $\theta \uparrow \infty$;

(a-ii) $g_{2}^{\prime}(\theta, 0) \uparrow \infty$ as $\theta \downarrow 0$ if $\eta / 2>\gamma_{2}$;

(a-iii) $g_{2}^{\prime}(\theta, 0) \uparrow-\frac{\phi_{2} \gamma_{2} \sigma^{2}}{\mu} e^{-\gamma_{2}\left(2 y_{R}-x\right)}$ as $\theta \downarrow 0$ if $\eta / 2=\gamma_{2}$;

(a-iv) $g_{2}^{\prime}(\theta, 0) \downarrow 0$ as $\theta \downarrow 0$ if $\eta / 2<\gamma_{2}$.

Let $\hat{\theta}^{(2)}=\hat{\theta}^{(2)}(0)$. If $\eta / 2 \geq \gamma_{2}$, the results above show that $\theta \mapsto g_{2}(\theta, 0)$ is concave for $\theta \in\left(0, \hat{\theta}^{(2)}\right]$ and $\theta \in\left[\hat{\theta}^{(2)}, \infty\right)$. Furthermore, it is also possible to show that 
$\lim _{\theta \uparrow \hat{\theta}^{(2)}} g_{2}^{\prime}(\theta, 0)<\lim _{\theta \downarrow \hat{\theta}^{(2)}} g_{2}^{\prime}(\theta, 0)$. From these two properties, we can conclude that $\bar{g}_{2}(\cdot, x)=g_{2}(\cdot, x)$ and hence the stopping region coincides again with all of $\mathcal{I}$, giving case $(\mathrm{v})$.

If $\eta / 2<\gamma_{2}$, (a-iv) implies that for constant $x, \theta \mapsto g_{2}(\theta, x)$ is convex for $\theta<\hat{\theta}^{(2)}(x)$ and concave otherwise. This implies that $\bar{g}_{2}(\theta, 0)$ is obtained by considering a chord from $\left(0,-\phi_{2}\right)$ to a point $\left(\bar{\theta}_{u}^{(2)}, g_{2}\left(\bar{\theta}_{u}^{(2)}, 0\right)\right)$ satisfying

$$
\frac{g_{2}\left(\bar{\theta}_{u}^{(2)}, 0\right)+\phi_{2}}{\bar{\theta}_{u}^{(2)}}=\left.\frac{\partial g_{2}}{\partial \theta}(\theta, 0)\right|_{\theta=\bar{\theta}_{u}^{(2)}},
$$

where $\bar{\theta}_{u}^{(2)} \geq \hat{\theta}^{(2)}$ since $\theta \mapsto g_{2}(\theta, 0)$ is convex over $\left(0, \hat{\theta}^{(2)}\right]$. Since $S^{-1}\left(\hat{\theta}^{(2)}\right)=y_{R}$, a similar argument to the proof of cases (ii) and (iii) of Proposition 3.3 gives

(b-i) $\bar{y}_{u}^{(2)}>y_{R}$ for $\frac{\phi_{1} \gamma_{2}}{\phi_{2}}>\frac{\eta}{2}$,

(b-ii) $\bar{y}_{u}^{(2)}=y_{R}$ for $\frac{\phi_{1} \gamma_{2}}{\phi_{2}} \leq \frac{\eta}{2}$,

where $\bar{y}_{u}^{(2)}=S^{-1}\left(\bar{\theta}_{u}^{(2)}\right)$. From (b-i) above, we see that when $\frac{\phi_{1} \gamma_{2}}{\phi_{2}}>\frac{\eta}{2}$, the investor first stops and sells at the level $\bar{y}_{u}^{(2)}>y_{R}$. However, since we are under the parameter regime where $\eta / 2<\gamma_{2} \leq \eta$ under which the stopping region of the second sale coincides with the whole space (as seen in the first part of the proof ${ }^{3}$ ), the investor then stops directly after selling the first unit of asset, giving case (iii)(a). A similar argument partially gives case (iv) from (b-ii) above. (In order to complete case (iv), it is also necessary to show that the investor also sells both assets at $y_{R}$ when $0<\eta<\gamma_{2}$; this case is considered in the next part of the proof below.)

Having gone through all the cases for $\eta \geq \gamma_{2}$, consider now the situation where $0<\eta<\gamma_{2}$ and suppose $\eta<\frac{\phi_{1} \gamma_{1}}{\phi_{2}}$. From the proof of Proposition 3.3, we know that under this parameter regime, $\bar{g}_{1}(\theta, x)$ is determined by a chord connecting $\left(0,-\phi_{2}\right)$ to $\left(\bar{\theta}_{u}, g_{1}\left(\bar{\theta}_{u}, x\right)\right)$ for $\theta \leq \bar{\theta}^{(1)}(x)$ and $\bar{g}_{1}(\theta, x)=g_{1}(\theta, x)$ otherwise. This gives

$$
\bar{g}_{1}(\theta, x)=\left\{\begin{array}{cc}
\phi_{1}\left(1-\exp \left(-\gamma_{1}\left(y_{R}-x\right)\right) \theta^{\gamma_{1} \frac{\sigma^{2}}{2 \mu}}\right) & \text { for } \theta \geq \bar{\theta}^{(1)}(x), \\
\left(\phi_{1}(1-\delta)+\phi_{2}\right) \delta^{-\left(2 \mu / \gamma_{1} \sigma^{2}\right)} & \\
\times \exp \left(\frac{2 \mu}{\sigma^{2}}\left(2 y_{R}-x\right)\right) \theta-\phi_{2} & \text { for } \left.\theta<\bar{\theta}^{(1)}(x)\right),
\end{array}\right.
$$

where

$$
\delta=\frac{\phi_{1}+\phi_{2}}{\phi_{1}} \frac{2 \mu}{2 \mu-\gamma_{1} \sigma^{2}}, \quad \bar{\theta}^{(1)}(x)=\exp \left(\frac{-2 \mu}{\sigma^{2}}\left(y_{R}-x\right)\right) \delta^{\left(2 \mu / \gamma_{1} \sigma^{2}\right)} .
$$

The solution to $V_{1}(y, x)$ under this parameter regime is described by the optimal stopping region $\Gamma=\left[\bar{y}_{u}^{(1)}, \infty\right)$, where $\bar{y}_{u}^{(1)}$ is given by (3.7). This can be observed from the results obtained in the proof of Proposition 3.3 (since $V_{1}(y, x)$ captures the same problem as described in Proposition 3.3). The next step is to use the transformation in

\footnotetext{
${ }^{3}$ Recall that we have $\tau_{2} \geq \tau_{1}$ and hence the problem described by the analysis of $g_{1}(\theta, x)$ relates to the second partial sale.
} 
(A.2) to solve for $V_{2}(y, x)$. By letting $g_{2}(\theta, x)=\bar{g}_{1}\left(\theta, x+S^{-1}(\theta)-y_{R}\right)$, we obtain

$$
g_{2}(\theta, x)= \begin{cases}\phi_{1}\left(1-\exp \left(-\gamma_{1}\left(2 y_{R}-x\right)\right) \theta^{\gamma_{1} \frac{\sigma^{2}}{\mu}}\right) & \text { for } \theta \geq \hat{\theta}^{(2)}(x), \\ K \exp \left(\frac{2 \mu}{\sigma^{2}}\left(2 y_{R}-x\right)\right) \theta^{2}-\phi_{2} & \text { for } \theta<\hat{\theta}^{(2)}(x),\end{cases}
$$

where

$$
K=\left(\phi_{1}(1-\delta)+\phi_{2}\right) \delta^{-\left(2 \mu / \gamma_{1} \sigma^{2}\right)}, \quad \hat{\theta}^{(2)}(x)=\exp \left(\frac{-\mu}{\sigma^{2}}\left(2 y_{R}-x\right)\right) \delta^{\left(\mu / \gamma_{1} \sigma^{2}\right)} .
$$

From our assumption that $\eta>0$, it is possible to show that $g_{2}^{\prime}(\theta, 0) \downarrow 0$ as $\theta \downarrow 0$ and $g_{2}^{\prime}(\theta, 0) \downarrow 0$ as $\theta \uparrow \infty$ and hence $g_{2}(\cdot, 0)$ is concave for $\theta>\hat{\theta}^{(2)}(x)$ and convex for $\theta<\hat{\theta}^{(2)}(x)$. The smallest concave majorant $\bar{g}_{2}(\cdot, 0)$ can be obtained by a chord joining two points on the graph of $g_{2}(\cdot, 0)$, replicating the same methodology as in the case above when $\eta / 2<\gamma_{2}<\eta$. The stopping region, which corresponds to the set $\left\{y \in \mathbb{R}: V_{1}(y, 0)=V_{2}(y, 0)\right\}=\left\{\theta>0: \bar{g}_{2}(\theta, 0)=g_{2}(\theta, 0)\right\}$, is given by $\left[\bar{y}_{u}^{(2)}, \infty\right)$, where

$$
\bar{y}_{u}^{(2)}=y_{R}-\frac{1}{2 \gamma_{1}} \ln \left(\frac{\phi_{1}+\phi_{2}}{\phi_{1}} \frac{\mu}{\mu-\gamma_{1} \sigma^{2}}\right) .
$$

Since $\eta<\phi_{1} \gamma_{1} / \phi_{2}$, it is in fact possible to show that $\bar{y}_{u}^{(2)}>y_{R}$.

This proves that the investor will sell the first asset at the level $\bar{y}_{u}^{(2)}$ and the second at any level at or above $\bar{y}_{u}^{(1)}$, proving case (ii). We now compare $\bar{y}_{u}^{(2)}$ and $\bar{y}_{u}^{(1)}$. It is straightforward to show that the condition

$$
\frac{\phi_{2}}{\phi_{1}} \frac{2 \eta}{\gamma_{1}}\left(1+\frac{\eta}{2 \gamma_{1}}\right)<1
$$

is equivalent to the ordering $\bar{y}_{u}^{(2)}<\bar{y}_{u}^{(1)}$, and hence in this case, the asset is sold at two distinct thresholds. If $\bar{y}_{u}^{(2)} \geq \bar{y}_{u}^{(1)}$, then both units are sold together at $\bar{y}_{u}^{(2)}$. This proves Corollary 3.5.

Finally, we examine the case when $0<\eta<\gamma_{2}$ and $\frac{\phi_{1} \gamma_{1}}{\phi_{2}} \leq \eta$. By using the characterisation of $\bar{g}_{1}(\cdot, x)$ obtained when proving Proposition 3.3 (iii) and using the relation described in (A.2), the following results can be obtained:

1) If $0<\eta<\gamma_{2}$ and $\frac{\eta}{2}<\frac{\gamma_{1} \phi_{1}}{\phi_{2}}<\eta$, the investor liquidates the first asset at the level $\bar{y}_{u}^{(2)}>y_{R}$, where $\bar{y}_{u}^{(2)}$ is given by (3.8), and the second asset at any level at or above $y_{R}$. This implies that both assets are sold at $\bar{y}_{u}^{(2)}$ (case (iii)(b)).

2) If $0<\eta<\gamma_{2}$ and $\frac{\gamma_{1} \phi_{1}}{\phi_{2}} \leq \frac{\eta}{2}$, the investor liquidates both assets at the reference level $y_{R}$. This completes the proof for case (iv).

\section{References}

1. Alvarez, L.H.R.: Singular stochastic control, linear diffusions, and optimal stopping: a class of solvable problems. SIAM J. Control Optim. 39, 1697-1710 (2001)

2. Alvarez, L.H.R.: On the properties of r-excessive mappings for a class of diffusions. Ann. Appl. Probab. 13, 1517-1533 (2003) 
3. Barberis, N.: A model of casino gambling. Manag. Sci. 58, 35-51 (2012)

4. Barberis, N., Xiong, W.: Realization utility. J. Financ. Econ. 104, 251-271 (2012)

5. Bayraktar, E., Egami, M.: On the one-dimensional optimal switching problem. Math. Oper. Res. 35, 140-159 (2010)

6. Carassus, L., Rásonyi, M.: On optimal investment for behavioural investors in discrete-time multiperiod incomplete markets. Math. Finance 25, 115-153 (2015)

7. Carmona, R., Dayanik, S.: Optimal multiple stopping of linear diffusions. Math. Oper. Res. 33, 446460 (2008)

8. Christensen, S.: On the solution of general impulse control problems using superharmonic functions. Stoch. Process. Appl. 124, 709-729 (2014)

9. Dayanik, S., Karatzas, I.: On the optimal stopping problem for one-dimensional diffusions. Stoch. Process. Appl. 107, 173-212 (2003)

10. Dynkin, E.B., Yushkevich, A.A.: Markov Processes: Theorems and Problems. Plenum Press, New York (1969)

11. Ebert, S., Strack, P.: Until the bitter end: on prospect theory in a dynamic context. Am. Econ. Rev. 105, 1618-1633 (2015)

12. Grasselli, M., Henderson, V.: Risk aversion and block exercise of executive stock options. J. Econ. Dyn. Control 33, 109-127 (2009)

13. He, X., Yang, L.: Realization utility with adaptive reference points. Math. Finance 29, 409-447 (2019)

14. Henderson, V.: Prospect theory, liquidation and the disposition effect. Manag. Sci. 58, 445-460 (2012)

15. Henderson, V., Hobson, D.: Optimal liquidation of derivative portfolios. Math. Finance 21, 365-382 (2011)

16. Henderson, V., Hobson, D., Tse, A.S.L.: Randomized strategies and prospect theory in a dynamic context. J. Econ. Theory 168, 287-300 (2017)

17. Henderson, V., Hobson, D., Tse, A.S.L.: Probability weighting, stop-loss and the disposition effect. J. Econ. Theory 178, 360-397 (2018)

18. Henderson, V., Sun, J., Whalley, A.E.: Portfolios of American options under general preferences: results and counterexamples. Math. Finance 24, 533-566 (2014)

19. Imas, A.: The realization effect: risk-taking after realized versus paper losses. Am. Econ. Rev. 106, 2086-2109 (2016)

20. Ingersoll, J.E., Jin, L.J.: Realization utility with reference dependent preferences. Rev. Financ. Stud. 26, 723-767 (2013)

21. Jin, H., Zhou, X.Y.: Behavioral portfolio selection in continuous time. Math. Finance 18, 385-426 (2008)

22. Kahneman, D., Tversky, A.: Prospect theory: an analysis of decision under risk. Econometrica 46, 171-185 (1979)

23. Kobylanski, M., Quenez, M.C., Rouy-Mironescu, E.: Optimal multiple stopping time problem. Ann. Appl. Probab. 21, 1365-1399 (2011)

24. Kyle, A.S., Ou-Yang, H., Xiong, W.: Prospect theory and liquidation decisions. J. Econ. Theory 129, 273-288 (2006)

25. Leung, T., Sircar, R.: Accounting for risk aversion, vesting, job termination risk and multiple exercises in valuation of employee stock options. Math. Finance 19, 99-128 (2009)

26. Lien, J., Zheng, J.: Deciding when to quit: reference-dependence over slot machine outcomes. Am. Econ. Rev. 115, 366-370 (2015)

27. Magnani, J.: Testing for the disposition effect on optimal stopping decisions. Am. Econ. Rev. 105, 371-375 (2015)

28. Odean, T.: Are investors reluctant to realize their losses? J. Finance 53, 1775-1798 (1998)

29. Peskir, G., Shiryaev, A.N.: Optimal Stopping and Free-Boundary Problems. Lectures in Mathematics, ETH Zürich. Birkhäuser, Basel (2006)

30. Revuz, D., Yor, M.: Continuous Martingales and Brownian Motion, 3rd edn. Springer, Berlin (1999)

31. Shefrin, H., Statman, M.: The disposition to sell winners too early and ride losers too long: theory and evidence. J. Finance 40, 777-790 (1985)

32. Thaler, R., Johnson, E.: Gambling with the house money and trying to break even: the effects of prior outcomes on risky choice. Manag. Sci. 36, 643-660 (1990)

33. Tversky, A., Kahneman, D.: Advances in prospect theory: cumulative representation of uncertainty. J. Risk Uncertain. 5, 297-323 (1992)

34. Xu, Z., Zhou, X.Y.: Optimal stopping under probability distortion. Ann. Appl. Probab. 23, 251-282 (2013) 